\title{
Pairwise Identity Verification via Linear Concentrative Metric Learning
}

\author{
Lilei Zheng, Student Member, IEEE, Stefan Duffner, Khalid Idrissi, Christophe Garcia, Atilla Baskurt
}

\begin{abstract}
This paper presents a study of metric learning systems on pairwise identity verification, including pairwise face verification and pairwise speaker verification, respectively. These problems are challenging because the individuals in training and testing are mutually exclusive, and also due to the probable setting of limited training data. For such pairwise verification problems, we present a general framework of metric learning systems and employ the stochastic gradient descent algorithm as the optimization solution. We have studied both similarity metric learning and distance metric learning systems, of either a linear or shallow nonlinear model under both restricted and unrestricted training settings. Extensive experiments demonstrate that with limited training pairs, learning a linear system on similar pairs only is preferable due to its simplicity and superiority, i.e. it generally achieves competitive performance on both the LFW face dataset and the NIST speaker dataset. It is also found that a pre-trained deep nonlinear model helps to improve the face verification results significantly.
\end{abstract}

Index Terms - metric learning, siamese neural networks, face verification, speaker verification, identity verification, pairwise metric

\section{INTRODUCTION}

$\mathrm{T}$ HE task of pairwise identity verification is to verify whether a pair of biometric identity samples corresponds to the same person or not, where the identity samples can be face images, speech utterances or any other biometric information from individuals. Formally, in such pairwise verification problems, two identity samples of the same person are called a similar pair, and two samples of two different persons are called a dissimilar pair or a different pair.

Compared with the traditional identity classification task in which a decision of acceptance or rejection is made by comparing an identity sample to models (or templates) of each individual [1], [2], [3], pairwise identity verification is more challenging because of the impossibility of building robust identity models with enough training data [4] for all the individuals. Actually, there may be only one identity sample available for some individuals in pairwise identity verification. Besides, individuals in training and testing should be mutually exclusive, i.e. the testing set comprises only samples from unknown persons that are not part of the training set.

Face images or speech utterances may be the most accessible and widely used identity information. As a result, face verification [1] and speaker verification [2] has been well studied over the last two decades. Especially, pairwise face verification has drawn much attention in recent years thanks to the popularity of the dataset 'Labeled Faces in the Wild' (LFW) [4]. Originally, the LFW dataset proposed a restricted training protocol where only a few specified data pairs are allowed for training, a challenging setting for

- All the authors are with Universite de Lyon, CNRS, INSA-Lyon, LIRIS, UMR5205, F-69621, France (e-mail: lilei.zheng@liris.cnrs.fr; lzheng@nwpu-aslp.org; khalid.idrissi@liris.cnrs.fr; la.baskurt@liris.cnrs.fr). stefan.duffner@liris.cnrs.fr christophe.garcia@liris.cnrs.fr; atil-

Manuscript received April 19, XXXX; revised September 17, XXXX. effective learning algorithms to discover principles from a small number of training examples, just like the human beings [5]. On the other hand, in the NIST Speaker Recognition Evaluations (SREs) since 1996, various speaker verification protocols have been investigated [6], [7]. In order to follow the pair generation scheme in the LFW standard protocol, we establish the pairwise speaker verification protocol based on the data from the NIST 2014 i-Vector Machine Learning Challenge [7].

The definition of pairwise identity verification reveals the need of measuring the difference or similarity between a pair of samples, which naturally leads us to the study of metric learning [8], i.e. methods that automatically learn a metric from a set of data pairs. A metric learning framework is implemented with a siamese architecture [9] which consists of two identical sub-systems sharing the same set of parameters. For a given input data pair, the two samples are processed by the two sub-systems respectively. The overall system includes a cost function parameterizing the pairwise relationship between data and a mapping function allowing the system to learn high-level features from the training data.

In terms of the cost function, one can divide metric learning methods into distance metric learning and similarity metric learning, where the cost function is defined based on a distance metric and a similarity measurement, respectively. The objective of such a cost function is to increase the similarity value or to decrease the distance between a similar pair, and to reduce the similarity value or to increase the distance between two dissimilar data samples. In this paper, we investigate two kinds of metric learning methods, namely, Triangular Similarity Metric Learning (TSML) [10] and Discriminative Distance Metric Learning (DDML) [11].

In terms of the mapping function, one can divide metric learning methods into two main families: linear metric learning and nonlinear metric learning. Up to now, work in metric learning has focused on linear methods because 
they are more convenient to optimize and less prone to over-fitting. For instance, the best approaches such as the Within Class Covariance Normalization (WCCN) and Cosine Similarity Metric Learning (CSML), have shown their effectiveness on the problem of pairwise face verification [12], [13]. Also, a few approaches have investigated nonlinear metric learning and have shown competitive performance on some classification problems [11], [14], [15]. Moreover, comparing linear systems with their nonlinear variants on a common ground helps to study the effect of nonlinearity on pairwise verification. For example, the nonlinear transformation - Diffusion Maps (DM) - has been introduced to face verification [13] and speaker verification [16], respectively. However, no clear evidence in the comparisons validated the universal effectiveness of DM over the linear systems [13]. Analogously, we present the TSML and DDML methods in both linear and nonlinear formulations for the sake of a thorough evaluation. Note that the nonlinear formulations are developed on the linear ones by adding nonlinear activation functions or stacking one more layer of transformation, thus the implemented nonlinearity is shallow.

Overall, on the problem of pairwise identity verification via metric learning, this paper presents a comprehensive study including two kinds of verification applications (i.e. face verification and speaker verification), two kinds of training settings (i.e. data-restricted and data-unrestricted), two kinds of metric learning cost functions (i.e. TSML and DDML), and three kinds of mapping functions (i.e. linear function, single-layer nonlinear function and multi-layer nonlinear function).

We will show that under the setting of limited training data, a linear metric learning system trained on similar pairs only generally yields competitive verification results. Either linear TSML or linear DDML achieves the state-of-the-art performance on both the LFW image dataset and the NIST speaker dataset.

The contributions of this paper with respect to previous works are the following:

- we establish a pairwise speaker verification protocol based on the data from the NIST 2014 i-Vector machine learning challenge, which has mutually exclusive training and test sets of speakers. Both the pairwise face verification protocol of the LFW dataset and this speaker verification task aim at verifying identity information by individuals' biometric features. Another objective of using the two datasets is to show the effectiveness of the proposed metric learning systems on different kinds of data, i.e. images and speech.

- we present the TSML and DDML methods in both linear and nonlinear formulations for pairwise identity verification problems. A thorough evaluation comparing the different formulations has shown that with limited training data, the linear models are preferable due to its superior performance and its simplicity.

- $\quad$ we study the influence of limited training data. Generally, compared with unlimited training, the limited case suffers from over-fitting. However, we find that training the linear models on similar pairs only considerably reduces the effect of over-fitting to limited training data.

- we also integrate the proposed linear and shallow nonlinear metric learning models with a pre-trained deep Convolutional Neural Network (CNN) model to improve the performance of pairwise face verification. We find that the linear model serves as an effective verification layer stacked to the deep CNN.

The remainder of this paper is organized as follows: Section 2 briefly summarizes the related work on metric learning and feature representations for images and speech. Section 3 presents the objective of metric learning by illustrating the cost functions of TSML and DDML. Section 4 introduces the linear and nonlinear formulations and explains the details of our stochastic gradient descent algorithm for optimization. Section 5 describes the datasets and experiments for pairwise face verification and pairwise speaker verification. Finally, we draw our conclusions in Section 6.

\section{Related Work}

\subsection{Metric Learning and Siamese Neural Networks}

Most of linear metric learning methods employ two types of metrics: the Mahalanobis distance or a more general similarity metric. In both of the two cases, a linear transformation matrix $W$ is learnt to project input features into a target space. Typically, distance metric learning concerns the Mahalanobis distance [17], [18]: $d_{W}(x, y)=\sqrt{(x-y)^{T} W(x-y)}$, where $x$ and $y$ are two sample vectors, and $W$ is the matrix that needs to be learnt. Note that when $W$ is the identity matrix, $d_{W}(x, y)$ is the Euclidean distance. In contrast, similarity metric learning methods learn a function of the following form: $s_{W}(x, y)=x^{T} W y / N(x, y)$, where $N(x, y)$ is a normalization term [19]. Specifically, when $N(x, y)=1, s_{W}(x, y)$ is the bilinear similarity function [20]; when $N(x, y)=\sqrt{x^{T} W x} \sqrt{y^{T} W y}, s_{W}(x, y)$ is the generalized cosine similarity function [12].

Nonlinear metric learning methods are constructed by simply substituting the above linear projection with a nonlinear transformation [11], [14], [15], [21]. For example, [11] and [14] employed neural networks to accomplish the nonlinear transformation. These nonlinear methods are subject to local optima and more inclined to over-fit to the training data but have the potential to outperform linear methods on some problems [8], [15]. Compared with linear models, nonlinear models are usually preferred on a redundant training set to well capture the underlying distribution of the data [22].

Since neural networks are the most commonly used nonlinear models, nonlinear metric learning has a natural connection with siamese neural networks [9], [14]. Actually, siamese neural networks can also be linear if the neurons have a linear activation function. From this point of view, siamese neural networks and metric learning denote the same technique of optimizing a metric-based cost function via a linear or nonlinear mapping. The difference exists in their names: "siamese neural networks" concern the symmetric structure of neural networks used for data mapping 
but the term "metric learning" emphasizes the pairwise relationship (i.e. the metric) in the data space.

For readers interested in a broader scope on metric learning in the literature, we recommend a recent survey which has provided an up-to-date and critical review of existing metric learning methods [8]. For those who prefer experimental analysis, an overview and empirical comparison is given in [23].

\subsection{Feature Representation for Face and Speaker}

For face recognition, tremendous efforts have been put on developing robust face descriptors [13], [24], [25], [26], [27], [28], [29], [30], [31], [32]. Popular face descriptors include eigenfaces [24], Gabor wavelets [27], SIFT [26], Local Binary Patterns (LBP) [25], etc. Especially, LBP and its variants, such as center-symmetric LBP (CSLBP) [33], multi-block LBP (MLLBP) [34], three patch LBP (TPLBP) [28] and over-complete LBP (OCLBP) [13], have been proven to be effective at describing facial texture. Especially, the high-dimensional variants usually perform better, for example, OCLBP [13]. Recently, another high-dimensional candidate, Fisher Vector (FV) face, which combines dense feature sampling with improved Fisher Vector encoding, has achieved striking results on pairwise face verification [30]. Besides, compared with the above handcrafted descriptors, automatical feature learning using Convolutional Neural Networks (CNN) has attracted a lot of interest in Computer Vision during the past decade [35], [36], [37]. In contrast to the handcrafted features, these CNN-based approaches usually rely on large training data to learn a lot of parameters, but they have substantially raised the state-of-the-art records on almost all the challenges in Computer Vision [38].

For speaker recognition, the most popular features are developed on generative models such as Gaussian Mixture Model-Universal Background Model (GMM-UBM) [39]. Building on the success of GMM-UBM, Joint Factor Analysis (JFA) proposes powerful tools to model the inter-speaker variability and to compensate for channel/session variability in the context of GMMs [40]. Moreover, inspired by JFA, a new feature called i-vector is developed [41], [42], [43]. JFA models the speaker variability in the high-dimensional space of GMM supervectors, whereas i-vectors are extracted in a low-dimensional space named total variability space. Taking advantage of the low dimensionality of the total variability space, many machine learning techniques can be applied to speaker verification [44]. Probabilistic Linear Discriminant Analysis (PLDA) [45] is one of the most popular techniques used for speaker verification: different variants such as the Gaussian PLDA (G-PLDA) [16], [46], HeavyTailed PLDA (HT-PLDA) [47], [48], [49] and Nonlinear PLDA [50] have been studied. In addition, Pairwise Support Vector Machines (PSVM) [51], [52] have been proposed to verify utterance pairs of different speakers; and fusing PSVM with PLDA can further improve the verification performance [46]. Recently, the metric learning framework DDML [11] was also shown to be helpful for PLDA-based speaker verification [50].

In our experiments, instead of studying the $\mathrm{CNN}$ for face verification or the PLDA for speaker verification, we focus on investigating the same metric learning models on the

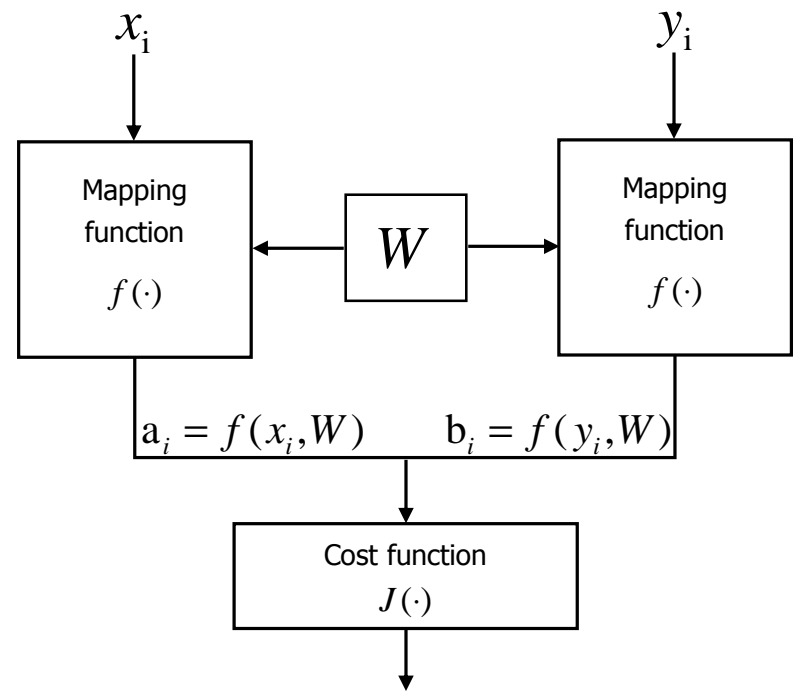

Attract similar pairs

Separate dissimilar pairs

Fig. 1. The siamese structure used in metric learning approaches. The objective is to find an optimal mapping, making a similar pair to be more closer and a dissimilar pair further apart.

two verification tasks. In terms of feature representations, we choose Fisher Vector faces as the face descriptors and i-vectors as the speech utterance descriptors.

\section{Metric Learning Objectives}

Metric learning algorithms usually employ the siamese architecture [9] to compare a pair of data inputs. Figure 1 shows the principal approach. A pair of data is given at the input, and two outputs are produced respectively with the current mapping function $f(\cdot)$. These outputs are constrained by a metric-based cost function $J(\cdot)$. By minimizing this cost function, we can achieve the objective of attracting similar pairs and separating dissimilar pairs. Concretely, if the pair of inputs are similar (i.e. from the same individual), the objective is to make the outputs more similar than the inputs; otherwise, the objective is to make the outputs more dissimilar/different. Popular choices of the measurement on the output vectors include the Euclidean distance [11], [18] and the Cosine Similarity [12], [20]. Therefore, we apply a distance metric learning method DDML [11] and a similarity metric learning method TSML [10] for the problem of pairwise identity verification.

By representing the face images or speech utterances as numerical vectors, we use a triplet $\left(x_{i}, y_{i}, s_{i}\right)$ to represent a pair of training input instances, where $x_{i}$ and $y_{i}$ are two vectors, and $s_{i}=1$ (respectively $s_{i}=-1$ ) means that the two vectors are similar (respectively dissimilar). Taking a projection $f(z, W)$ on the inputs, we obtain a new pair $\left(a_{i}, b_{i}\right)$ in the target space, where $a_{i}=f\left(x_{i}, W\right)$ and $b_{i}=f\left(y_{i}, W\right)$. Then, the TSML or DDML cost function is constructed to define the pairwise relationship between $a_{i}$ and $b_{i}$. Finally, the procedure of learning the metric is carried out by minimizing the cost on a set of training pairs.

\subsection{Triangular Similarity Metric Learning}

TSML concerns the Triangular Similarity which is equivalent to the Cosine Similarity [53]. On the two outputs $a_{i}$ and 


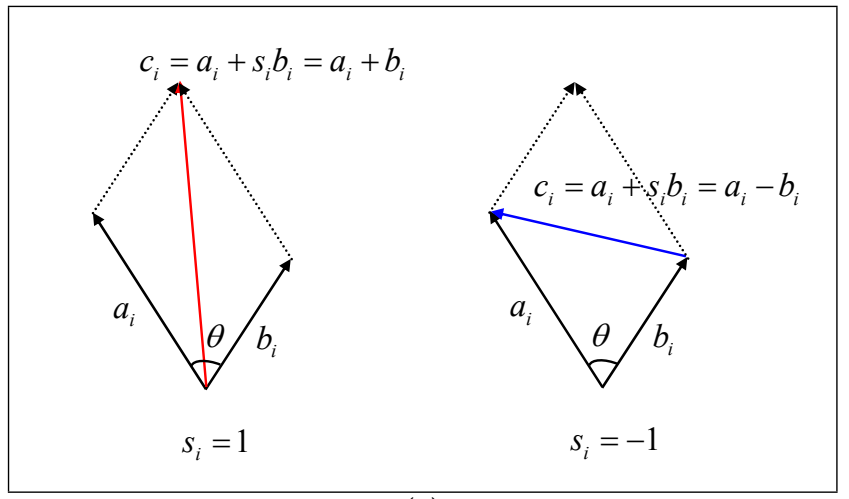

(a)

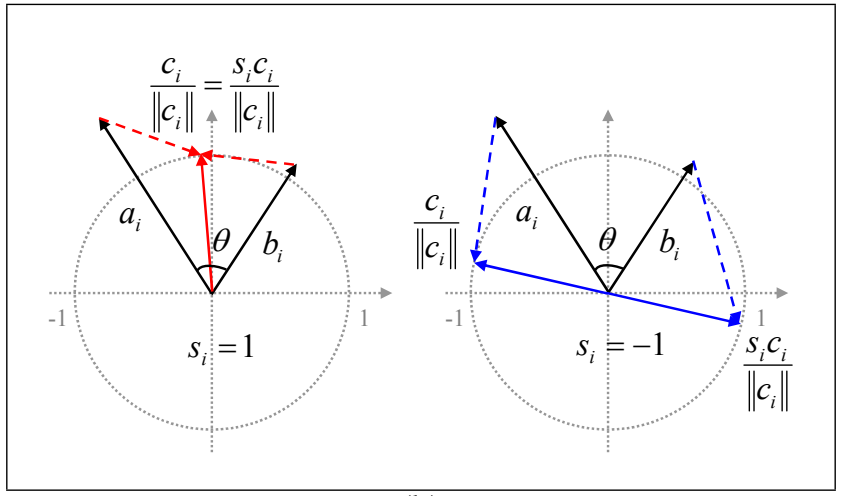

(b)

Fig. 2. Geometrical interpretation of the TSML cost and gradient. (a) Minimizing the cost means to make similar vectors parallel and make dissimilar vectors opposite. (b) The gradient function suggests unit vectors on the diagonals as targets for $a_{i}$ and $b_{i}$ : the same target vector for a similar pair $\left(s_{i}=1\right)$; or the opposite target vectors for a dissimilar pair $\left(s_{i}=-1\right)$.

$b_{i}$, the cost function of TSML is defined as:

$$
J_{i}=\frac{1}{2}\left\|a_{i}\right\|^{2}+\frac{1}{2}\left\|b_{i}\right\|^{2}-\left\|c_{i}\right\|+1
$$

where $c_{i}=a_{i}+s_{i} b_{i}: c_{i}$ can be regarded as one of the two diagonals of the parallelogram formed by $a_{i}$ and $b_{i}$ (Fig. 2(a)). Moreover, this cost function can be rewritten as:

$$
J_{i}=\frac{1}{2}\left(\left\|a_{i}\right\|-1\right)^{2}+\frac{1}{2}\left(\left\|b_{i}\right\|-1\right)^{2}+\left\|a_{i}\right\|+\left\|b_{i}\right\|-\left\|c_{i}\right\| .
$$

We can see that minimizing the first part aims to make the vectors $a_{i}$ and $b_{i}$ having unit length 1 ; the second part concerns the well-known triangle inequality theorem: the sum of the lengths of two sides of a triangle must always be greater than the length of the third side, i.e. $\left\|a_{i}\right\|+\left\|b_{i}\right\|-\left\|c_{i}\right\|>0$. More interestingly, with the length constraints by the first part, minimizing the second part is equivalent to minimizing the angle $\theta$ inside a similar pair $\left(s_{i}=1\right)$ or maximizing the angle $\theta$ inside a dissimilar pair $\left(s_{i}=-1\right)$, in other words, minimizing the Cosine Similarity between $a_{i}$ and $s_{i} b_{i}$ :

$$
\cos \left(a_{i}, s_{i} b_{i}\right)=s_{i} \frac{a_{i}^{T} b_{i}}{\left\|a_{i}\right\|\left\|b_{i}\right\|} .
$$

The gradient of the cost function (Equation (1)) with respect to the parameters $W$ is:

$$
\frac{\partial J_{i}}{\partial W}=\left(a_{i}-\frac{c_{i}}{\left\|c_{i}\right\|}\right)^{T} \frac{\partial a_{i}}{\partial W}+\left(b_{i}-\frac{s_{i} c_{i}}{\left\|c_{i}\right\|}\right)^{T} \frac{\partial b_{i}}{\partial W} .
$$

We can obtain the optimal cost at the zero gradient: $a_{i}-\frac{c_{i}}{\left\|c_{i}\right\|}=0$ and $b_{i}-\frac{s_{i} c_{i}}{\left\|c_{i}\right\|}=0$. In other words, the gradient function has $\frac{c_{i}}{\left\|c_{i}\right\|}$ and $\frac{c_{i} c_{i}}{\left\|c_{i}\right\|}$ as targets for $a_{i}$ and $b_{i}$, respectively. Fig. 2(b) illustrates that: for a similar pair, $a_{i}$ and $b_{i}$ are mapped to the same target vector along the diagonal (the red solid line); for a dissimilar pair, $a_{i}$ and $b_{i}$ are mapped to opposite unit vectors along the other diagonal (the blue solid line). This perfectly reveals the objective of attracting similar pairs and separating dissimilar pairs.

\subsection{Discriminative Distance Metric Learning}

In contrast, DDML focuses on the pairwise distance between feature vectors. Unlike the Cosine Similarity naturally defines a minimum of -1 and a maximum of 1 , the Euclidean

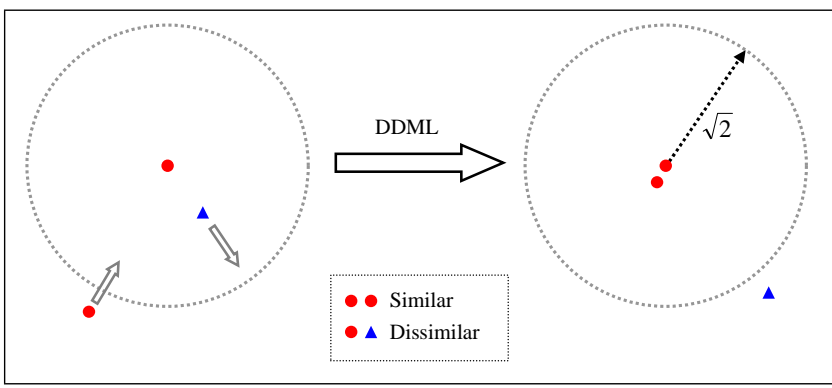

Fig. 3. Illustration of the DDML cost function, whose objective is to find an optimal mapping to make a similar pair closer and to separate a dissimilar pair with a distance margin of $\sqrt{2}$.

distance has only a minimum of 0 and no maximum. Hence a margin is usually defined in distance metric learning to assume that two vectors with a distance larger than the margin are well separated.

Typically, for a pair of outputs $a_{i}$ and $b_{i}$, DDML defines the cost function as:

$$
J_{i}=\frac{1}{2} g\left(1-s_{i}\left(1-\left(a_{i}-b_{i}\right)^{2}\right)\right),
$$

where $g(z)=\frac{1}{T} \log (1+\exp (T z))$ is the generalized logistic loss function [54], $T$ is a sharpness parameter usually set to 10. Minimizing the logistic loss function means to minimize the value of

$$
z_{i}=1-s_{i}\left(1-\left(a_{i}-b_{i}\right)^{2}\right) .
$$

Specifically, for a similar pair $\left(s_{i}=1\right), z_{i}$ can be simplified as $\left(a_{i}-b_{i}\right)^{2}$, and minimizing $z_{i}$ requires $a_{i}$ and $b_{i}$ to be identical; for a dissimilar pair $\left(s_{i}=-1\right)$, the equation suggests maximizing $-z_{i}=\left(a_{i}-b_{i}\right)^{2}-2$, that is to separate a dissimilar pair with a distance of $\sqrt{2}$. An illustration of the objective is shown in Fig. 3.

The gradient of the DDML cost function (Equation (5)) with respect to the parameters $W$ is:

$$
\frac{\partial J_{i}}{\partial W}=\frac{s_{i}\left(a_{i}-b_{i}\right)}{1+\exp \left(-T\left(1-s_{i}+s_{i}\left(a_{i}-b_{i}\right)^{2}\right)\right)} \frac{\partial\left(a_{i}-b_{i}\right)}{\partial W} .
$$

\subsection{Cost and Gradient for Batch Training}

In practice, we may consider a few data pairs as a small batch in each training iteration, thus the overall cost and 
gradient of a batch is simply the average from all the training pairs in the batch:

$$
\begin{gathered}
J=\frac{1}{n} \sum_{i=1}^{n} J_{i}, \\
\frac{\partial J}{\partial W}=\frac{1}{n} \sum_{i=1}^{n} \frac{\partial J_{i}}{\partial W},
\end{gathered}
$$

where $n$ is the number of training pairs in a batch, $J_{i}$ is the TSML cost in Equation (1) or the DDML cost in Equation (5), the corresponding gradient $\frac{\partial J_{i}}{\partial W}$ is calculated by Equation (4) or Equation (7). Finally, the gradient can be used in the Backpropagation algorithm [55] to perform gradient descent and search an optimal solution.

\section{Linear and Nonlinear Mappings}

When a cost function defines the pairwise relationship between data in the target space, a mapping function represents the system's ability of learning to achieve the goal of the cost function. From the point of view of neural networks, different mapping functions can be considered as different combinations of neurons in network layers. We study three kinds of mapping functions here:

\section{Single layer of linear neurons}

The simplest neurons are the linear neurons without bias term which only involve a parameter matrix $W$. For a given input $z \in R^{d}$, the output is simply $f(z, W)=W z$. For instance, the TSML gradient of the $i_{t h}$ pair with respect to the parameter matrix $W$ is:

$$
\frac{\partial J_{i}}{\partial W}=\left(a_{i}-\frac{c_{i}}{\left\|c_{i}\right\|}\right) x_{i}^{T}+\left(b_{i}-s_{i} \frac{c_{i}}{\left\|c_{i}\right\|}\right) y_{i}^{T} .
$$

\section{Single layer of nonlinear neurons}

Besides the parameter matrix $W$, nonlinear neurons involve a bias term, and a nonlinear activation function, e.g. the tanh function [22]. For a given input $z \in R^{d}$, the output is:

$$
f(z, W)=\tanh (W z+h),
$$

where $h$ denotes the bias term of the neurons. This equation can be rewritten as:

$$
f\left(z^{\prime}, W^{\prime}\right)=\tanh \left(W^{\prime} z^{\prime}\right),
$$

where $z^{\prime}=[z ; 1]$ and $W^{\prime}=\left[\begin{array}{ll}W & h\end{array}\right]$. Remind that derivative of the tanh function is $\tanh ^{\prime}(z)=1-\tanh ^{2}(z)$. Based on the linear case in Equation (9), the derivative of the TSML cost function with respect to the parameters $W^{\prime}:\{W, h\}$ is:

$$
\begin{aligned}
\frac{\partial J_{i}}{\partial W^{\prime}} & =\left(a_{i}-\frac{c_{i}}{\left\|c_{i}\right\|}\right) \frac{\partial a_{i}}{\partial W^{\prime}}+\left(b_{i}-\frac{s_{i} c_{i}}{\left\|c_{i}\right\|}\right) \frac{\partial b_{i}}{\partial W^{\prime}} \\
& =\left[\left(1-a_{i} \odot a_{i}\right) \odot\left(a_{i}-\frac{c_{i}}{\left\|c_{i}\right\|}\right)\left[x_{i} ; 1\right]^{T}\right. \\
& \left.+\left(1-b_{i} \odot b_{i}\right) \odot\left(b_{i}-\frac{s_{i} c_{i}}{\left\|c_{i}\right\|}\right)\left[y_{i} ; 1\right]^{T}\right],
\end{aligned}
$$

where the notation $\odot$ means element-wise multiplication. The derivation of this equation can be easily obtained with the chain rule used in the Backpropagation algorithm [22].

\section{Multiple layers of nonlinear neurons}

By combining several interconnected nonlinear neurons together, Multi-Layer Perceptrons (MLP) are able to approximate arbitrary nonlinear mappings and thus have been the most popular kind of neural networks since the 1980's [55]. We adopt a 3-layer MLP, containing one input layer and two layers of nonlinear neurons, to realize the nonlinear mapping.

Similar with Equation (12) and according to the Backpropagation chain rule, we can calculate derivatives with respect to each parameter of the MLP for a given training pair.

For the DDML cost function, we can obtain derivatives with respect to the weights of neuron layers in the same way with the TSML method. For all the linear and shallow nonlinear systems, we employ the same stochastic gradient descent optimization to update their weights until reaching an optimal solution.

\subsection{Stochastic Gradient Descent}

Since all the three types of mapping functions have similar cost and gradient functions, we employ the same algorithm to perform optimization. The proposed method is based on stochastic gradient descent and is summarized in Algorithm 1. More advanced optimization algorithms such as conjugate gradient descent, L-BFGS [10], [56] could be used as well but their analysis would go beyond the scope of this paper. We adopt early-stopping [57] to prevent the over-fitting problem. Thus a small set is separated from the training data for validation, and the model with the best performance on the validation set is retained for evaluation on the test set. In addition, we use a momentum [22] term to speed up training. The momentum $\lambda$ is empirically set to be 0.99 for all the experiments. Following [30], [58], the input vectors will be passed through L2 normalization before training, i.e. the length of input vectors are normalized to 1.

\section{Initializing the weights}

For the linear mapping, like in [10], [12], [58], we initialize the transformation matrix with the identity matrix. For the nonlinear mappings, we use the normalized random initialization [59] that is considered to be helpful for the tanh networks. Concretely, weights of each layer are initialized with an uniform distribution as:

$$
\left\{W^{(j)}, h^{(j)}\right\} \sim U\left[-\frac{\sqrt{6}}{\sqrt{n_{j}+n_{j+1}}}, \frac{\sqrt{6}}{\sqrt{n_{j}+n_{j+1}}}\right],
$$

where $\left\{W^{(j)}, h^{(j)}\right\}$ denotes the parameters between the $j_{t h}$ and $(j+1)_{t h}$ layers; $n_{j}$ and $n_{j+1}$ represent the number of nodes in the two layers, respectively.

\section{EXPERIMENTS AND ANALYSIS}

\subsection{Datasets}

In order to validate the generality of the proposed approaches, we carried out pairwise identity verification experiments on two datasets in different domains: the LFW image dataset for pairwise face verification [4] and the NIST i-vector dataset for pairwise speaker verification [7]. 
TABLE 1

Distribution of individuals and images in the 10 subsets, where the individuals are mutually exclusive.

\begin{tabular}{|c||c|c|c|c|c|c|c|c|c|c|c|}
\hline Index & 1 & 2 & 3 & 4 & 5 & 6 & 7 & 8 & 9 & 10 & Total \\
\hline \hline Number of individuals & 601 & 555 & 552 & 560 & 567 & 527 & 597 & 601 & 580 & 609 & 5749 \\
\hline Number of images & 1369 & 1367 & 1089 & 1324 & 1016 & 1166 & 1690 & 1222 & 1207 & 1783 & 13233 \\
\hline
\end{tabular}

TABLE 2

Distribution of individuals and speech utterances in the 10 subsets, where the individuals are mutually exclusive.

\begin{tabular}{|c||c|c|c|c|c|c|c|c|c|c|c|}
\hline Index & 1 & 2 & 3 & 4 & 5 & 6 & 7 & 8 & 9 & 10 & Total \\
\hline \hline Number of individuals & 496 & 496 & 496 & 496 & 496 & 496 & 496 & 496 & 496 & 494 & 4958 \\
\hline Number of utterances & 3660 & 3664 & 3568 & 3741 & 3702 & 3566 & 3605 & 3636 & 3744 & 3686 & 36572 \\
\hline
\end{tabular}

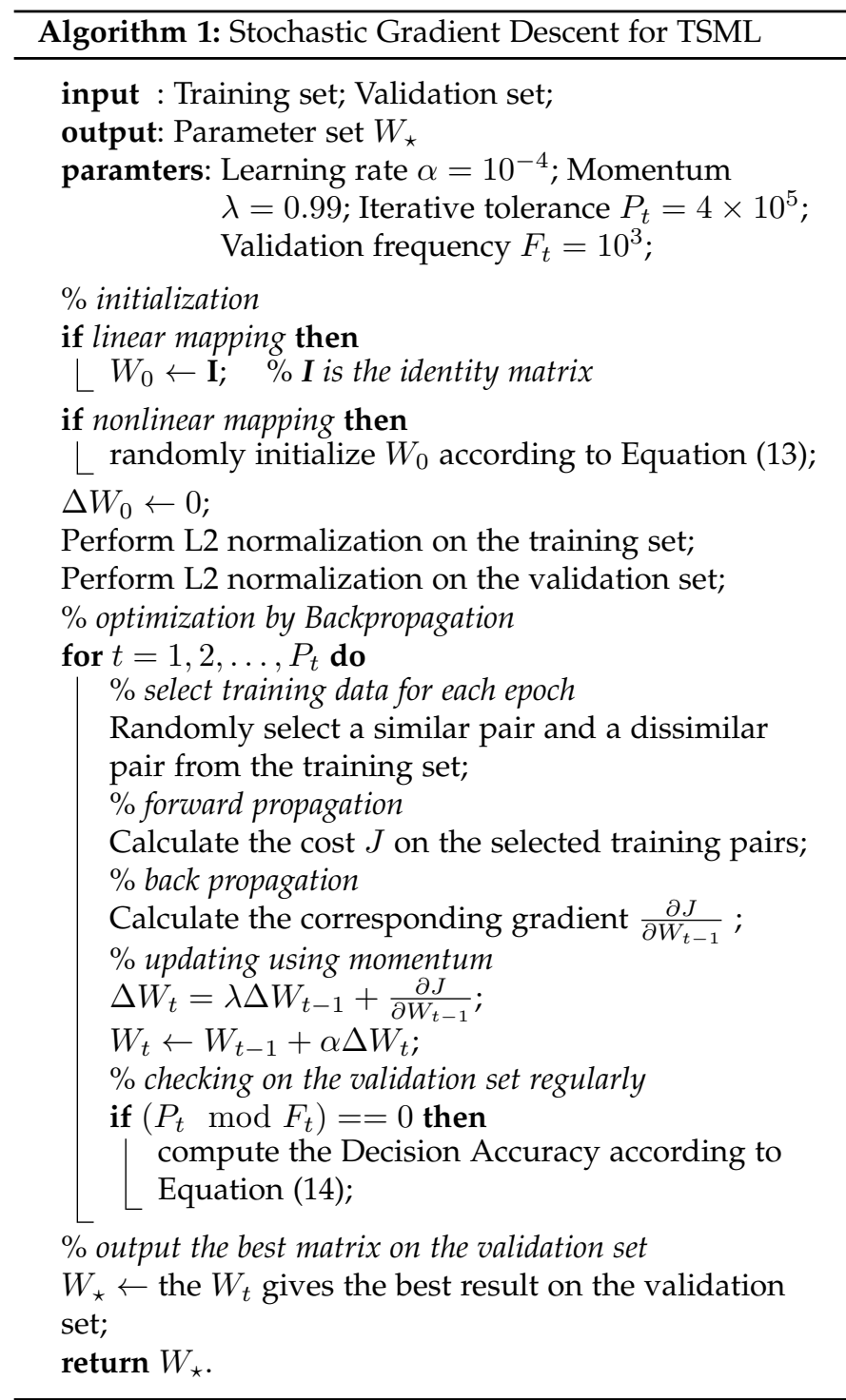

\subsubsection{LFW dataset}

The LFW dataset contains numerous annotated images from the web. For all the images, we used the cropped $150 \times 150$ 'funneled' version of LFW [4]. We only used the View 2 subset of LFW for performance evaluation. In View 2, to do 10 -fold cross validation, all the 5749 persons in the dataset are divided into 10 subsets where the individuals are mutually exclusive. The total number of images for all the persons is 13,233 , however, the number of images for each individual varies from 1 to 530 . Table 1 summarizes the data distribution of individuals and images in the 10 subsets.

We used Fisher Vector faces as vector representation of face images, where data of the vectors are directly provided by $[30]^{1}$ (Data for the setting 3 ), and the dimension of a Fisher Vector face is 67,584 . However, directly taking the original facial vectors for learning causes computational problems, i.e. the time required for multiplications of the $67,584-\mathrm{d}$ vectors would be unacceptable. Therefore, following [12], [13], we apply Whitened Principal Component Analysis (WPCA) to reduce the vector dimension to 500 .

\subsubsection{NIST i-vector dataset}

We used the data of the NIST 2014 Speaker i-Vector Challenge [7], which consist of i-vectors derived from conversational telephone speech data in the NIST speaker recognition evaluations from 2004 to 2012. Each i-vector, the identity vector, is a vector of 600 components. Along with each i-vector, the amount of speech (in seconds) used to compute the i-vector is supplied as metadata. Segment durations were sampled from a log normal distribution with a mean of 39.58 seconds. This dataset consists of a development set for building models and a test set for evaluation.

We only used the development data of this Challenge and established an experimental protocol of pairwise speaker verification. There are 36,572 speech utterances in total in this experiment, belonging to 4958 different speakers. The number of utterances for a single speaker varies from 1 to 75. Like in LFW, we also split the data into 10 subsets to do 10 -fold cross validation. Table 2 shows the distribution of individuals and speech utterances in the 10 subsets.

\subsection{Experimental Setup}

On both of the two datasets, we performed cross-validation on the 10 folds: there are overall 10 experiments, in each repetition, sample pairs from 9 folds are used for training, and sample pairs from the remaining fold are used for testing. As we have announced in Section 4.1, some training data are separated as an independent validation set to do early-stopping.

\subsubsection{Fixed testing}

To perform evaluation on the test set for each experiment, it is better to fix the sample pairs in each fold so that we can fairly compare different approaches on the same test data. Specifically, 600 image pairs are provided in each fold of the

1. http://www.robots.ox.ac.uk/〜vgg/software/face_desc/ 
LFW dataset, where 300 are similar and the other 300 are dissimilar [4]. In the NIST i-vector dataset, there are more samples for each individual than in the LFW dataset, so we generate more sample pairs for each fold, namely, 1200 similar pairs and 1200 dissimilar pairs.

\subsubsection{Restricted and unrestricted training}

Following [4], we defined two training settings in our experiments: the restricted setting in which only the fixed sample pairs in each fold can be collected for training, e.g. the specified 300 similar and 300 dissimilar pairs in each fold of the LFW dataset; in contrast, the unrestricted setting allows to generate more sample pairs for training by using the identity information of all the samples. As mentioned previously, the test sample pairs are the same for both restricted and unrestricted settings.

\subsubsection{Maximal decision accuracy}

Like the minimal Decision Cost Function (minDCF) in [7], we define a Decision Accuracy (DA) function to measure the overall verification performance on a set of data pairs:

$$
D A(\gamma)=\frac{\text { number of right decisions }(\gamma)}{\text { total number of pairs }} \text {, }
$$

where the threshold $\gamma$ is used to make a decision on the final distance or similarity values: for the TSML system, $\cos (a, b)>\gamma$ means $(a, b)$ is a similar pair, otherwise it is dissimilar; for the DDML system, $(a-b)^{2}<\gamma$ denotes a similar pair, otherwise it is dissimilar. The maximal DA (maxDA) over all possible threshold values is the final score recorded. We report the mean maxDA scores ( \pm standard error of the mean) of the 10 experiments. For the speaker verification results, we also measure the mean Equal Error Rate (EER) as it is commonly used in the speaker recognition field [47], [52].

\subsection{Experimental Results}

At the beginning, we directly calculated maxDA scores on the whitened feature vectors, i.e. the 500-dimensional FV vectors for the LFW dataset and 600-dimensional i-vectors for the NIST i-vector dataset. We consider this evaluation as the baseline. According to the different neuron models defined in Section 4, we evaluated three kinds of metric learning approaches in the experiments:

- TSML-Linear and DDML-Linear: using a single layer of linear neurons without bias term;

- TSML-Nonlinear and DDML-Nonlinear: using a single layer of nonlinear neurons with a bias term;

- TSML-MLP and DDML-MLP: using two layers of nonlinear neurons with bias terms;

All these models are trained on both similar and dissimilar pairs. Results on the LFW-funneled dataset and the NIST i-vector dataset are summarized in Tables 3-6. We also reimplement the state-of-the-art WCCN method [13], [58] as a comparison.

Learning on Similar Pairs Only: comparing WCCN with the proposed six metric learning models, we find that WCCN achieves better performance under the restricted training. The major difference between WCCN and the other
TABLE 3

Mean maxDA scores ( \pm standard error of the mean) of pairwise face verification by the TSML systems on the LFW-funneled image dataset. '-Sim' means learning on similar pairs only.

\begin{tabular}{ccc}
\hline Approaches & Restricted Training & Unrestricted Training \\
\hline Baseline & \multicolumn{2}{c}{$84.83 \pm 0.38$} \\
WCCN & $91.10 \pm 0.45$ & $91.17 \pm 0.36$ \\
\hline TSML-Linear & $87.95 \pm 0.40$ & $92.03 \pm 0.38$ \\
TSML-Nonlinear & $86.23 \pm 0.39$ & $91.43 \pm 0.52$ \\
TSML-MLP & $84.10 \pm 0.45$ & $89.30 \pm 0.73$ \\
\hline TSML-Linear-Sim & $\mathbf{9 1 . 9 0} \pm \mathbf{0 . 5 2}$ & $\mathbf{9 2 . 4 0} \pm \mathbf{0 . 4 8}$ \\
TSML-Nonlinear-Sim & $90.58 \pm 0.52$ & $91.47 \pm 0.37$ \\
TSML-MLP-Sim & $88.98 \pm 0.64$ & $89.03 \pm 0.58$ \\
\hline
\end{tabular}

TABLE 4

Mean maxDA scores ( \pm standard error of the mean) and mean EER of pairwise speaker verification by the TSML systems on the NIST i-vector speaker dataset. '-Sim' means learning on similar pairs only.

\begin{tabular}{ccc}
\hline Approaches & Restricted Training & Unrestricted Training \\
\hline Baseline & \multicolumn{2}{c}{$87.78 \pm 0.39 / 0.1335$} \\
WCCN & $91.69 \pm 0.29 / 0.0900$ & $91.97 \pm 0.33 / 0.0853$ \\
\hline TSML-Linear & $89.78 \pm 0.25 / 0.1108$ & $93.97 \pm 0.20 / \mathbf{0 . 0 6 4 8}$ \\
TSML-Nonlinear & $87.43 \pm 0.31 / 0.1340$ & $93.11 \pm 0.20 / 0.0733$ \\
TSML-MLP & $84.88 \pm 0.24 / 0.1592$ & $90.21 \pm 0.36 / 0.1023$ \\
\hline TSML-Linear-Sim & $\mathbf{9 2 . 9 4} \pm \mathbf{0 . 1 5} / \mathbf{0 . 0 7 8 5}$ & $\mathbf{9 3 . 9 9} \pm \mathbf{0 . 2 4} / 0.0662$ \\
TSML-Nonlinear-Sim & $\mathbf{9 1 . 2 9} \pm 0.25 / 0.0918$ & $93.43 \pm 0.23 / 0.0690$ \\
TSML-MLP-Sim & $89.59 \pm 0.45 / 0.1093$ & $90.83 \pm 0.30 / 0.0967$ \\
\hline
\end{tabular}

models is that WCCN concerns only intra-personal variance but ignores the inter-personal information [13], [58]. In other words, WCCN performs learning on similar pairs only but the current TSML and DSML systems take into account both similar and dissimilar pairs. To clarify this issue, we train the proposed models on similar pairs only as six new models: TSML-Linear-Sim, TSML-Nonlinear-Sim and TSML-MLPSim; DDML-Linear-Sim, DDML-Nonlinear-Sim and DDMLMLP-Sim. The results are also shown in Tables $3-6$.

\subsubsection{More training data}

The first phenomenon we can observe is that unrestricted training produces better results than restricted training. More training data generally bring up an accuracy improvement to each model. We have known since mid-seventies [5], [38], [60] that many methods increase in accuracy with increasing training data until they reach optimal performance. Indeed, more training data better capture the underlying distribution of the whole dataset and thus reduce the overfitting gap between training and test. Especially for the pairwise verification problem that requires learning on data pairs, compared with restricted training only allows to use a few specified training pairs in a dataset, unrestricted training covers enough data pairs and thus protect the models from over-fitting to a small portion of training data.

\subsubsection{Linear vs. nonlinear}

The second observation is that the linear models generally perform better than the shallow nonlinear models. Specifically, more parameters (i.e. additional bias terms or/and more layers of neurons) and the nonlinearity make the 
TABLE 5

Mean maxDA scores ( \pm standard error of the mean) of pairwise face verification by the DDML systems on the LFW-funneled image dataset. '-Sim' means learning on similar pairs only.

\begin{tabular}{ccc}
\hline Approaches & Restricted Training & Unrestricted Training \\
\hline Baseline & \multicolumn{2}{c}{$84.83 \pm 0.38$} \\
WCCN & $\mathbf{9 1 . 1 0} \pm \mathbf{0 . 4 5}$ & $91.17 \pm 0.36$ \\
\hline DDML-Linear & $88.27 \pm 0.53$ & $\mathbf{9 2 . 4 8} \pm \mathbf{0 . 3 5}$ \\
DDML-Nonlinear & $88.12 \pm 0.70$ & $92.23 \pm 0.36$ \\
DDML-MLP & $88.60 \pm 0.90$ & $91.53 \pm 0.42$ \\
\hline DDML-Linear-Sim & $91.03 \pm 0.61$ & $91.80 \pm 0.29$ \\
DDML-Nonlinear-Sim & $90.82 \pm 0.45$ & $91.42 \pm 0.40$ \\
DDML-MLP-Sim & $89.57 \pm 0.45$ & $89.53 \pm 0.44$ \\
\hline
\end{tabular}

TABLE 6

Mean maxDA scores ( \pm standard error of the mean) and mean EER of pairwise speaker verification by the DDML systems on the NIST i-vector speaker dataset. '-Sim' means learning on similar pairs only.

\begin{tabular}{clc}
\hline Approaches & Restricted Training & Unrestricted Training \\
\hline Baseline & \multicolumn{2}{c}{$87.78 \pm 0.39 / 0.1335$} \\
WCCN & $91.69 \pm 0.29 / 0.0900$ & $91.97 \pm 0.33 / 0.0853$ \\
\hline DDML-Linear & $89.77 \pm 0.21 / 0.1127$ & $94.32 \pm 0.23 / 0.0612$ \\
DDML-Nonlinear & $87.98 \pm 0.29 / 0.1262$ & $93.36 \pm 0.23 / 0.0703$ \\
DDML-MLP & $89.11 \pm 0.27 / 0.1143$ & $92.39 \pm 0.25 / 0.0807$ \\
\hline DDML-Linear-Sim & $\mathbf{9 2 . 9 5} \pm \mathbf{0 . 2 9} / \mathbf{0 . 0 7 4 8}$ & $\mathbf{9 4 . 4 2} \pm \mathbf{0 . 2 4} / \mathbf{0 . 0 5 9 0}$ \\
DDML-Nonlinear-Sim & $91.98 \pm 0.25 / 0.0850$ & $93.74 \pm 0.22 / 0.0662$ \\
DDML-MLP-Sim & $89.08 \pm 0.27 / 0.1133$ & $89.69 \pm 0.36 / 0.1075$ \\
\hline
\end{tabular}

nonlinear models more powerful to adapt themselves to the training data. However, without any additional techniques to prevent over-fitting, generalization to the test data is not guaranteed. Figure 4 shows the learning curves of TSMLLinear, TSML-Nonlinear and TSML-MLP in restricted training, we can see that all of them easily fit the training data. Especially, with the most parameters, TSML-MLP is the strongest learning machine that reaches the accuracy of $100 \%$ on the training data with the fewest iterations, but it performs the worst on the test data. More regularization techniques, such as weight decay [22] and dropout [61], can be introduced to reduce the risk of over-fitting for such slightly deeper nonlinear model, but their analysis would go beyond the scope of this paper. In contrast, with the same experimental setting, linearity naturally indicates the property of generalization and thus makes TSML-Linear better fit to the unseen data, i.e. the validation and test sets.

\subsubsection{Concentrative training on limited data pairs}

Figure 5 compares the performance of the linear models of both TSML and DDML on the LFW-funneled dataset and the NIST i-vector datset, respectively. In general, under the restricted training, the models trained on similar pairs only, i.e. TSML-Linear-Sim and DDML-Linear-Sim, yield significantly better results; under the unrestricted training, all the linear models perform comparably well.

In general, a linear concentrative model $^{2}$ should be adopted for restricted training because of its superior per-

2. We use the term "concentrative" to indicate learning on similar pairs only since it concerns closing a similar pair rather than separating a dissimilar pair.

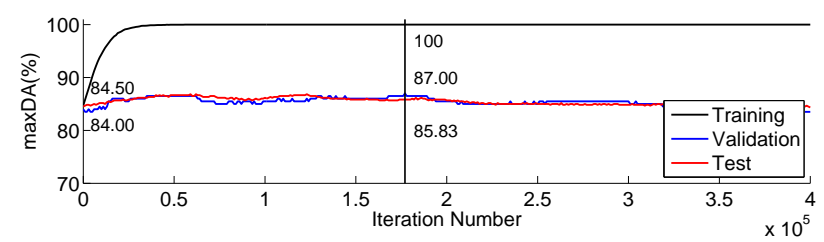

(a) Learning curve of TSML-Linear

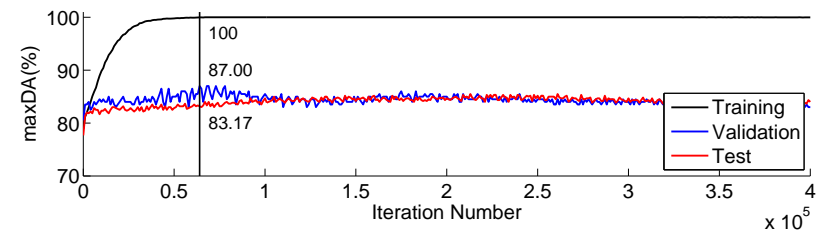

(b) Learning curve of TSML-Nonlinear

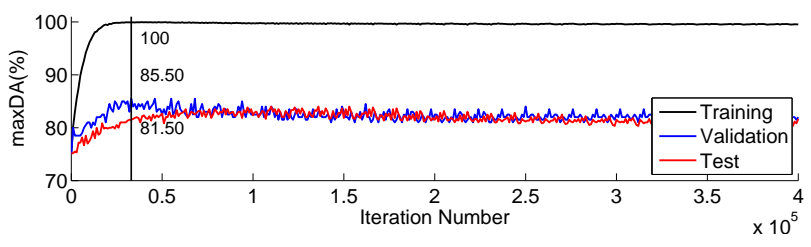

(c) Learning curve of TSML-MLP

Fig. 4. Learning curves of different TSML models. Curves on the training, validation and test sets are represented by black, blue and red lines, respectively. All the models are trained on the LFW data under the restricted setting. According to early stopping, the vertical line indicates the model having the best performance on the validation set. Without any additional regularization techniques, the more complex the learning model is, i.e. having more parameters, the larger the over-fitting gap is.

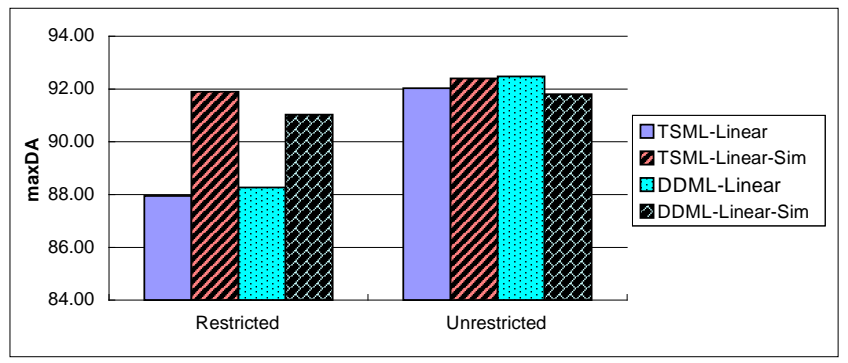

(a) Results on LFW-funneled

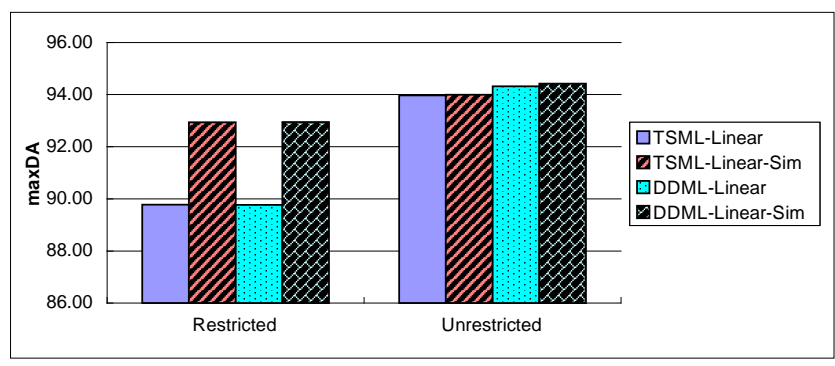

(b) Results on NIST i-vector

Fig. 5. Performance comparison of the linear models on the LFWfunneled dataset and the NIST i-vector dataset.

formance. Moreover, it should be also preferred for unrestricted training due to faster training. Compared with models trained on both similar and dissimilar pairs, the linear concentrative models only take into account half of the training data but yield comparable verification results.

Concretely, the setting of equal quantity of similar and dissimilar pairs is problematic for restricted training. As- 
suming a $n$-class problem with two samples in each class, the number of all possible similar pairs is $n$. But the number of all possible dissimilar pairs is $2 n(n-1)$, which is much larger than the number of similar pairs. However, the restricted configuration requires the number of dissimilar pairs is the same as the number of similar pairs. For example, only 300 similar pairs and 300 dissimilar pairs are provided in each subset of the LFW dataset. As a consequence, learning on such limited number of dissimilar pairs causes serious over-fitting problems to the normal models, that is why they perform worse than the linear concentrative models. In contrast, when the training is unrestricted, enough dissimilar pairs can be covered during training and the risk of over-fitting is reduced. Hence the normal models trained on both similar and dissimilar pairs perform well in unrestricted training.

In short, restricted training on equal quantity of similar and dissimilar pairs does not accord with the ratio of similar and dissimilar pairs in practice. The similar pairs indeed deliver more positive contributions for learning a better metric. Apart from our suggestion of learning on similar pairs only, this goal can be achieved by other techniques such as shifting the Cosine Similarity boundary [62], using hinge loss functions to filter invalid gradient descent from dissimilar pairs [11] or weighting the gradient contributions from similar and dissimilar pairs [12], [63]. Overall, our proposed concentrative training is a competitive choice due to its simplicity.

\subsubsection{TSML vs. DDML}

Comparing the two metric learning methods, TSML and DDML, we find comparable performance records in Tables 3 - 6. This is reasonable because the Euclidean distance is naturally related to the Cosine Similarity. For the square of the Euclidean distance between two vectors, we have $(a-b)^{2}=(a-b)^{T}(a-b)=a^{2}+b^{2}-2 a^{T} b$. When the vectors are normalized to unit length, i.e. $a^{2}=b^{2}=1$, the previous equation can be written as $(a-b)^{2}=2-2 \cos (a, b)$. That means in our situation, minimizing the distance between data pairs is equivalent to maximizing the pairwise similarity value.

\subsection{Comparison with the State-of-the-Art}

We compared the proposed TSML-Linear-Sim method with several state-of-the-art methods on the LFW dataset under the image-restricted configuration with no outside data [64]. The comparison is summarized in Table 7, and the corresponding ROC curves are shown in Fig. 6. The curves of MRF-MLBP [65] and MRF-Fusion-CSKDA [66] are missing because the curve data are not provided on the public result page ${ }^{3}$. We can see that MRF-Fusion-CSKDA occupies the first place and the proposed TSML-Linear-Sim takes the second one with a relatively large gap $(91.90 \%$ vs. $95.89 \%$ ). This is because MRF-Fusion-CSKDA employed multi-scale binarized statistical image features and made a fusion on multiple features [66]. However, the proposed TSML-Linear-Sim method is much simpler as it has only utilized a single feature, the FV vectors.

3. http://vis-www.cs.umass.edu/lfw/results.htmlfImageRestrictedNo
TABLE 7

Comparison of TSML-Linear-Sim with other state-of-the-art results under the restricted configuration with no outside data on LFW-funneled.

\begin{tabular}{cc}
\hline Method & Accuracy \\
\hline V1-like/MKL [67] & $79.35 \pm 0.55$ \\
APEM (fusion) [68] & $79.06 \pm 1.51$ \\
MRF-MLBP [65] (no ROC) & $79.08 \pm 0.14$ \\
SVM-Fisher vector faces [30] & $87.47 \pm 1.49$ \\
Eigen-PEP (fusion) [69] & $88.97 \pm 1.32$ \\
Hierarchical-PEP (fusion) [70] & $91.10 \pm 1.47$ \\
MRF-Fusion-CSKDA [66] (no ROC) & $\mathbf{9 5 . 8 9} \pm \mathbf{1 . 9 4}$ \\
\hline TSML-Linear-Sim (this work) & $\mathbf{9 1 . 9 0} \pm \mathbf{0 . 5 2}$ \\
\hline
\end{tabular}

TABLE 8

Comparison of TSML-Linear-Sim with other methods using single face descriptor under the restricted configuration with no outside data on LFW-funneled.

\begin{tabular}{ccc}
\hline Method & Feature & Accuracy \\
\hline MRF-MLBP [65] & multi-scale LBP & $79.08 \pm 0.14$ \\
APEM [68] & SIFT & $81.88 \pm 0.94$ \\
APEM [68] & LBP & $81.97 \pm 1.90$ \\
Eigen-PEP [69] & PEP & $88.47 \pm 0.91$ \\
Hierarchical-PEP [70] & PEP & $90.40 \pm 1.35$ \\
SVM [30] & Fisher Vector faces & $87.47 \pm 1.49$ \\
DDML-Linear-Sim & Fisher Vector faces & $91.03 \pm 0.61$ \\
WCCN [13] & Fisher Vector faces & $91.10 \pm 0.45$ \\
\hline TSML-Linear-Sim & Fisher Vector faces & $\mathbf{9 1 . 9 0} \pm \mathbf{0 . 5 2}$ \\
\hline
\end{tabular}

Thus we collected the results of methods using a single feature in Table 8. Especially, we also applied another stateof-the-art approach WCCN [13] on the FV vectors as a comparison. We can see that the proposed TSML-Linear-Sim method achieves the best performance (91.90\%) among all the methods using a single feature. Especially, TSML-LinearSim significantly surpasses the conventional Support Vector Machines (SVM) method [30] on the FV vectors by $4.43 \%$ points (from $87.47 \%$ to $91.90 \%$ ).

\subsection{Stacked to Pre-trained Deep Nonlinearity}

As we have mentioned, the proposed shallow nonlinearity was constrained due to lack of proper generalization strategies and more training data. At present, the success of deep learning in speech recognition and visual object recognition shows that the deep nonlinearity is able to learn discriminative representations of data [38]. To release the power of nonlinearity, deep learning approaches require large datasets and perform training in a supervised way [35], [36], [37]. However, it is difficult to directly train a deep metric learning system on a large dataset having hundred thousands of or even millions of data samples [35], [71] because the number of sample pairs will be dramatically raised. Actually training semi-supervised siamese neural networks is much slower than training supervised neural networks [53]. Recent empirical work showed that training siamese neural networks on carefully chosen triplets instead of data pairs is helpful for fast convergence [72], [73].

Besides, it was also found that even a simple classifier can make good decision on the features produced by the o learned deep models [35], [36], [71]. Therefore we stack 


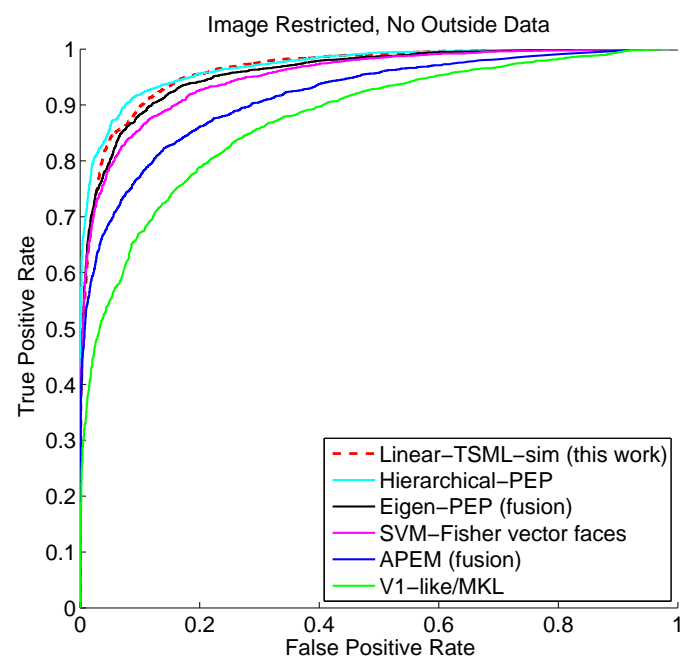

Fig. 6. ROC curves of Linear-TSML-Sim (red dashed line) and other state-of-the-art methods on the LFW dataset under the restricted configuration with no outside data.

TABLE 9

Mean maxDA scores ( \pm standard error of the mean) of pairwise face verification by stacking the metric learning systems to the pre-trained deep CNN model on the LFW-funneled image dataset.

\begin{tabular}{cccc}
\hline Approaches & \multicolumn{3}{c}{ Accuracy } \\
\hline Deep CNN & \multicolumn{3}{c}{$97.93 \pm 0.22$} \\
\hline \hline -Linear & -Nonlinear & -MLP \\
\hline Deep CNN-TSML & $\mathbf{9 8 . 2 5} \pm \mathbf{0 . 1 9}$ & $97.50 \pm 0.21$ & $97.15 \pm 0.22$ \\
Deep CNN-DDML & $\mathbf{9 8 . 1 8} \pm \mathbf{0 . 2 2}$ & $97.78 \pm 0.26$ & $97.20 \pm 0.26$ \\
\hline
\end{tabular}

the proposed linear and nonlinear metric learning models to a pre-trained deep CNN [71] trained on the CASISWebface dataset [74]. There are 493,456 labeled images of 10,575 identities in the CASIS-Webface. [71] provides two deep models trained on these data. We use the model A to extract features from each face image in the LFW dataset, resulting in a 256-dimensional vector. Then the process of metric learning is similar with that on the Fisher Vectors under the unrestricted training setting. All the TSML and DDML models are tested.

Table 9 summarizes the results of the deep CNN mod$\mathrm{el}$ and the stacked models. It is not surprising that the deep CNN brings significant verification improvement to our shallow models. By the learned discriminative feature representations from the CASIS-Webface face images, the deep CNN itself achieves the accuracy of $97.93 \%$. We can see that the linear models, TSML-Linear and DDML-Linear, further improve the verification performance to $98.25 \%$ and $98.18 \%$. This improvement is guaranteed by the identity initialization and early stopping applied to the linear models: the deep CNN results are taken as initial status for metric learning; and early stopping marks the best record on the validation set. In contrast, the shallow nonlinear metric learning models obtain slightly worse results because they take random initialization and degrade the good deep CNN baseline. A probable reason is that we have restricted the input/output size of the nonlinear models to the size of the linear models, and it might be possible to improve the nonlinear models by tuning the size of layers, trying different initialization methods or adding regularization techniques. However, the simple linear metric learning model is indeed a good and quick option that demands less effort on hyperparameter tuning than the shallow nonlinear ones. Thus we suggest the deep nonlinearity for robust feature learning on large datasets and the shallow linearity for classification [37].

\section{Conclusion}

In this paper, we have evaluated two metric learning methods - TSML and DDML - for pairwise face verification on the LFW dataset and pairwise speaker verification on the NIST i-vector dataset. Under the setting of limited training pairs, we found that learning a linear model on similar pairs only is a simple but effective solution for identify verification. When labeled outside data are available, a pre-trained deep CNN model helps the linear TSML and DDML systems to reach competitive performance on face verification.

We presented several strategies and confirmed their effectiveness on reducing the risk of over-fitting. These strategies include using more training pairs; using a linear model to keep generalization; learning on similar pairs only for restricted training; separating a validation set to perform early stopping; introducing a deep CNN model pre-trained on a large dataset. With these strategies, the nature of learning a good metric of the TSML and DDML methods makes themselves effective on the two different pairwise verification tasks.

The defined pairwise verification task is not limited to only human identities, the objects can be documents, audio, images or individuals in any other categories. For any pairwise verification problems with objects that can be represented as numerical vectors, we believe that the proposed methods are applicable, and the observed phenomena are repeatable.

\section{ACKNOWLEDGMENTS}

Thanks to the China Scholarship Council (CSC) and the Laboratoire d'InfoRmatique en Image et Systèmes d'information (LIRIS) for supporting this work.

\section{REFERENCES}

[1] J. Matas, M. Hamouz, K. Jonsson, J. Kittler, Y. Li, C. Kotropoulos, A. Tefas, I. Pitas, T. Tan, H. Yan, et al., "Comparison of face verification results on the XM2VTFS database," in Proc. ICPR. IEEE, 2000, vol. 4, pp. 858-863.

[2] D. A. Reynolds, "Speaker identification and verification using gaussian mixture speaker models," Speech communication, vol. 17, no. 1, pp. 91-108, 1995.

[3] Dapeng Tao, Lianwen Jin, Yongfei Wang, and Xuelong Li, "Person reidentification by minimum classification error-based kiss metric learning," IEEE transactions on cybernetics, vol. 45, no. 2, pp. 242252,2015

[4] G. B. Huang, M. Ramesh, T. Berg, and E. Learned-Miller, "Labeled faces in the wild: A database for studying face recognition in unconstrained environments," Tech. Rep., University of Massachusetts, Amherst, 2007.

[5] Erik Learned-Miller, Gary Huang, Aruni RoyChowdhury, Haoxiang Li, and Gang Hua, "Labeled faces in the wild: A survey," 2015. 
[6] Alvin F Martin and Craig S Greenberg, "The NIST 2010 speaker recognition evaluation," in Eleventh Annual Conference of the International Speech Communication Association, 2010.

[7] C. S. Greenberg, D. Bansé, G. R. Doddington, D. Garcia-Romero, J. J. Godfrey, T. Kinnunen, A. F. Martin, A. McCree, M. Przybocki, and D. A. Reynolds, "The NIST 2014 speaker recognition i-vector machine learning challenge," in Odyssey: The Speaker and Language Recognition Workshop, 2014.

[8] A. Bellet, A. Habrard, and M. Sebban, "A survey on metric learning for feature vectors and structured data," Computing Research Repository, vol. abs/1306.6709, 2013.

[9] J. Bromley, J. W. Bentz, L. Bottou, I. Guyon, Y. LeCun, C. Moore, E. Säckinger, and R. Shah, "Signature verification using a Siamese time delay neural network," International Journal of Pattern Recognition and Artificial Intelligence, vol. 7, no. 04, pp. 669-688, 1993.

[10] L. Zheng, K. Idrissi, C. Garcia, S. Duffner, and A. Baskurt, "Triangular similarity metric learning for face verification," in Proc. FG, 2015.

[11] J. Hu, J. Lu, and Y.-P. Tan, "Discriminative deep metric learning for face verification in the wild," in Proc. CVPR, 2014, pp. 1875-1882.

[12] N. V. Hieu and B. Li, "Cosine similarity metric learning for face verification," in Proc. ACCV. 2011, pp. 709-720, Springer.

[13] O. Barkan, J. Weill, L. Wolf, and H. Aronowitz, "Fast high dimensional vector multiplication face recognition," in Proc. ICCV, 2013.

[14] S. Chopra, R. Hadsell, and Y. LeCun, "Learning a similarity metric discriminatively, with application to face verification," in Proc. CVPR. IEEE, 2005, vol. 1, pp. 539-546.

[15] D. Kedem, S. Tyree, F. Sha, G. R. Lanckriet, and K. Q. Weinberger, "Non-linear metric learning," in Advances in Neural Information Processing Systems, 2012, pp. 2573-2581.

[16] Oren Barkan and Hagai Aronowitz, "Diffusion maps for PLDAbased speaker verification," in 2013 IEEE International Conference on Acoustics, Speech and Signal Processing. IEEE, 2013, pp. 76397643.

[17] E. P. Xing, A. Y. Ng, M. I. Jordan, and S. Russell, "Distance metric learning with application to clustering with side-information," Advances in Neural Information Processing Systems, pp. 521-528, 2003.

[18] K. Weinberger, J. Blitzer, and L. Saul, "Distance metric learning for large margin nearest neighbor classification," Advances in Neural Information Processing Systems, vol. 18, pp. 1473, 2006.

[19] A. M. Qamar, E. Gaussier, J. P. Chevallet, and J. H. Lim, "Similarity learning for nearest neighbor classification," in Proc. ICDM. IEEE, 2008, pp. 983-988.

[20] G. Chechik, V. Sharma, U. Shalit, and S. Bengio, "Large scale online learning of image similarity through ranking," The Journal of Machine Learning Research, vol. 11, pp. 1109-1135, 2010.

[21] Jun Yu, Xiaokang Yang, Fei Gao, and Dacheng Tao, "Deep multimodal distance metric learning using click constraints for image ranking.," IEEE transactions on cybernetics, 2016.

[22] Y. A. LeCun, L. Bottou, G. B. Orr, and K.-R. Müller, “Efficient backprop," in Neural networks: Tricks of the trade, pp. 9-48. Springer, 2012.

[23] Panagiotis Moutafis, Mengjun Leng, and Ioannis A Kakadiaris, "An overview and empirical comparison of distance metric learning methods," IEEE Transactions on Cybernetics, 2016.

[24] Ma. A. Turk and A. P. Pentland, "Face recognition using eigenfaces," in Proc. CVPR. IEEE, 1991, pp. 586-591.

[25] T. Ahonen, A. Hadid, and M. Pietikäinen, "Face recognition with local binary patterns," in Proc. ECCV. 2004, pp. 469-481, Springer.

[26] Y. Ke and R. Sukthankar, "PCA-SIFT: A more distinctive representation for local image descriptors," in Proc. CVPR. IEEE, 2004, vol. 2, pp. II-506.

[27] J. G. Daugman, "Complete discrete 2-D gabor transforms by neural networks for image analysis and compression," Acoustics, Speech and Signal Processing, IEEE Transactions on, vol. 36, no. 7, pp. $1169-1179,1988$.

[28] L. Wolf, T. Hassner, and Y. Taigman, "Descriptor based methods in the wild," in Workshop on Faces in'Real-Life'Images: Detection, Alignment, and Recognition, 2008.

[29] S. U. Hussain, T. Napoléon, and F. Jurie, "Face recognition using local quantized patterns," in Proc. BMVC, 2012.

[30] K. Simonyan, O. M. Parkhi, A. Vedaldi, and A. Zisserman, "Fisher vector faces in the wild," in Proc. BMVC, 2013, vol. 1, p. 7.
[31] D. Chen, X. Cao, F. Wen, and J. Sun, "Blessing of dimensionality: High-dimensional feature and its efficient compression for face verification," in Proc. CVPR. IEEE, 2013, pp. 3025-3032.

[32] Chuan-Xian Ren, Zhen Lei, Dao-Qing Dai, and Stan Z Li, “Enhanced local gradient order features and discriminant analysis for face recognition," IEEE Transactions on Cybernetics, vol. PP, no. 99, pp. 1-14, 2015.

[33] M. Heikkilä, M. Pietikäinen, and C. Schmid, "Description of interest regions with center-symmetric local binary patterns," in Computer Vision, Graphics and Image Processing, pp. 58-69. Springer, 2006.

[34] L. Zhang, R. Chu, S. Xiang, S. Liao, and S. Z. Li, "Face detection based on multi-block lbp representation," in Advances in biometrics, pp. 11-18. Springer, 2007.

[35] Y. Taigman, M. Yang, M. A. Ranzato, and L. Wolf, "Deepface: Closing the gap to human-level performance in face verification," in Proc. CVPR. IEEE, 2014, pp. 1701-1708.

[36] Y. Sun, Y. Chen, X. Wang, and X. Tang, "Deep learning face representation by joint identification-verification," in Advances in Neural Information Processing Systems, 2014, pp. 1988-1996.

[37] K. Chatfield, K. Simonyan, A. Vedaldi, and A. Zisserman, "Return of the devil in the details: Delving deep into convolutional nets," in Proc. BMVC, 2014.

[38] Yann A LeCun, Yoshua Bengio, and Geoffrey E Hinton, “Deep learning," Nature, vol. 521, pp. 436-444, 2015.

[39] D. A. Reynolds, T. F. Quatieri, and R. B. Dunn, "Speaker verification using adapted Gaussian mixture models," Digital signal processing, vol. 10, no. 1, pp. 19-41, 2000.

[40] P. Kenny, P. Ouellet, N. Dehak, V. Gupta, and P. Dumouchel, "A study of interspeaker variability in speaker verification," Audio, Speech, and Language Processing, IEEE Transactions on, vol. 16, no. 5, pp. 980-988, 2008.

[41] N. Dehak, P. Kenny, R. Dehak, P. Dumouchel, and P. Ouellet, "Front-end factor analysis for speaker verification," Audio, Speech, and Language Processing, IEEE Transactions on, vol. 19, no. 4, pp. 788-798, 2011.

[42] Daniel Garcia-Romero and Carol Y Espy-Wilson, "Analysis of ivector length normalization in speaker recognition systems.," in Interspeech, 2011, pp. 249-252.

[43] Yun Lei, Nicolas Scheffer, Luciana Ferrer, and Mitchell McLaren, "A novel scheme for speaker recognition using a phoneticallyaware deep neural network," in 2014 IEEE International Conference on Acoustics, Speech and Signal Processing (ICASSP). IEEE, 2014, pp. 1695-1699.

[44] A. Larcher, K. A. Lee, B. Ma, and H. Li, "Text-dependent speaker verification: Classifiers, databases and RSR2015," Speech Communication, vol. 60, pp. 56-77, 2014.

[45] Simon JD Prince and James H Elder, "Probabilistic linear discriminant analysis for inferences about identity," in 2007 IEEE 11th International Conference on Computer Vision. IEEE, 2007, pp. 1-8.

[46] Sibel Yaman and Jason Pelecanos, "Using polynomial kernel support vector machines for speaker verification," IEEE Signal Processing Letters, vol. 20, no. 9, pp. 901-904, 2013.

[47] Patrick Kenny, "Bayesian speaker verification with heavy-tailed priors," in Odyssey, 2010, p. 14.

[48] Lukáš Burget, Oldřich Plchot, Sandro Cumani, Ondřej Glembek, Pavel Matějka, and Niko Brümmer, "Discriminatively trained probabilistic linear discriminant analysis for speaker verification," in 2011 IEEE international conference on acoustics, speech and signal processing (ICASSP). IEEE, 2011, pp. 4832-4835.

[49] Pavel Matějka, Ondřej Glembek, Fabio Castaldo, Md Jahangir Alam, Oldřich Plchot, Patrick Kenny, Lukáš Burget, and Jan Černocky, "Full-covariance UBM and heavy-tailed PLDA in ivector speaker verification," in 2011 IEEE International Conference on Acoustics, Speech and Signal Processing (ICASSP). IEEE, 2011, pp. $4828-4831$.

[50] Sergey Novoselov, Timur Pekhovsky, Oleg Kudashev, Valentin Mendelev, and Alexey Prudnikov, "Non-linear PLDA for i-vector speaker verification," ISCA Interspeech, 2015.

[51] Sandro Cumani, Niko Brümmer, Lukáš Burget, and Pietro Laface, "Fast discriminative speaker verification in the i-vector space," in 2011 IEEE International Conference on Acoustics, Speech and Signal Processing (ICASSP). IEEE, 2011, pp. 4852-4855.

[52] Sandro Cumani and Pietro Laface, "Large-scale training of pairwise support vector machines for speaker recognition," IEEE/ACM Transactions on Audio, Speech and Language Processing (TASLP), vol. 22, no. 11, pp. 1590-1600, 2014. 
[53] Lilei Zheng, Triangular Similarity Metric Learning: a Siamese Architecture Approach, Ph.D. thesis, University of Lyon, 2016.

[54] Alexis Mignon and Frédéric Jurie, "Pcca: A new approach for distance learning from sparse pairwise constraints," in Computer Vision and Pattern Recognition (CVPR), 2012 IEEE Conference on. IEEE, 2012, pp. 2666-2672.

[55] David E Rumelhart, Geoffrey E Hinton, and Ronald J Williams, "Learning internal representations by error propagation," Tech. Rep., DTIC Document, 1985.

[56] D. C. Liu and J. Nocedal, "On the limited memory BFGS method for large scale optimization," Mathematical programming, vol. 45, no. 1-3, pp. 503-528, 1989.

[57] Lutz Prechelt, "Early stopping - but when?," in Neural Networks: Tricks of the Trade, pp. 53-67. Springer, 2012.

[58] Q. Cao, Y. Ying, and P. Li, "Similarity metric learning for face recognition," in Proc. ICCV, 2013.

[59] Xavier Glorot and Yoshua Bengio, "Understanding the difficulty of training deep feedforward neural networks," in International conference on artificial intelligence and statistics, 2010, pp. 249-256.

[60] Charles J Stone, "Consistent nonparametric regression," The annals of statistics, pp. 595-620, 1977.

[61] Nitish Srivastava, Geoffrey Hinton, Alex Krizhevsky, Ilya Sutskever, and Ruslan Salakhutdinov, "Dropout: A simple way to prevent neural networks from overfitting," The Journal of Machine Learning Research, vol. 15, no. 1, pp. 1929-1958, 2014.

[62] Lilei Zheng, Khalid Idrissi, Christophe Garcia, Stefan Duffner, and Atilla Baskurt, "Logistic similarity metric learning for face verification," in Acoustics, Speech and Signal Processing, 2015 IEEE International Conference on. IEEE, 2015.

[63] Junlin Hu, Jiwen Lu, and Yap-Peng Tan, “Deep transfer metric learning," in Proceedings of the IEEE Conference on Computer Vision and Pattern Recognition, 2015, pp. 325-333.

[64] G. B. Huang and E. Learned-Miller, "Labeled faces in the wild: Updates and new reporting procedures,"

[65] S. R. Arashloo and J. Kittler, "Efficient processing of MRFs for unconstrained-pose face recognition," in Biometrics: Theory, Applications and Systems (BTAS), 2013 IEEE Sixth International Conference on. IEEE, 2013, pp. 1-8.

[66] Shervin Rahimzadeh Arashloo and Josef Kittler, "Class-specific kernel fusion of multiple descriptors for face verification using multiscale binarised statistical image features," Information Forensics and Security, IEEE Transactions on, vol. 9, no. 12, pp. 2100-2109, 2014.

[67] N. Pinto, J. J. DiCarlo, and D. D. Cox, "How far can you get with a modern face recognition test set using only simple features?," in Proc. CVPR. IEEE, 2009, pp. 2591-2598.

[68] H. Li, G. Hua, Z. Lin, J. Brandt, and J. Yang, "Probabilistic elastic matching for pose variant face verification," in Proc. CVPR. IEEE, 2013, pp. 3499-3506.

[69] Haoxiang Li, Gang Hua, Xiaohui Shen, Zhe Lin, and Jonathan Brandt, "Eigen-pep for video face recognition," in Computer Vision-ACCV 2014, pp. 17-33. Springer, 2015.

[70] H. Li and G. Hua, "Hierarchical-PEP model for real-world face recognition," in Proc. CVPR. 2015, pp. 4055-4064, IEEE.

[71] Xiang Wu, Ran He, and Zhenan Sun, "A lightened CNN for deep face representation," arXiv preprint arXiv:1511.02683, 2015.

[72] Florian Schroff, Dmitry Kalenichenko, and James Philbin, "Facenet: A unified embedding for face recognition and clustering," in Proceedings of the IEEE Conference on Computer Vision and Pattern Recognition, 2015, pp. 815-823.

[73] Omkar M Parkhi, Andrea Vedaldi, and Andrew Zisserman, "Deep face recognition," in British Machine Vision Conference, 2015, vol. 1, p. 6.

[74] Dong Yi, Zhen Lei, Shengcai Liao, and Stan Z Li, "Learning face representation from scratch," arXiv preprint arXiv:1411.7923, 2014.

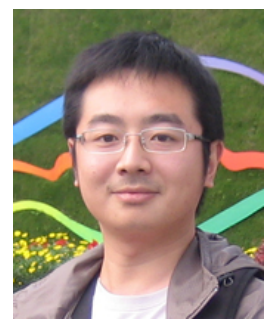

Lilei Zheng received a Bachelor's degree in 2009 and a Master's degree in 2012, all in computer science and technology from Northwestern Polytechnical University, Xi'an, China. He is currently a Ph.D. student in the school of in formation and mathematics, University of Lyon. His current research interests include machine learning and computer vision.

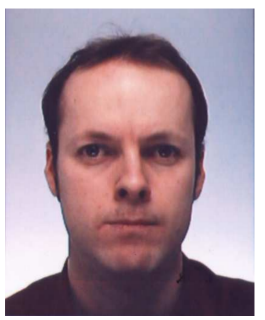

Stefan Duffner received a Bachelor's degree in Computer Science from the University of $A p-$ plied Sciences Konstanz, Germany in 2002 and a Master's degree in Applied Computer Science from the University of Freiburg, Germany in 2004. He performed his dissertation research at Orange Labs in Rennes, France, on face image analysis with statistical machine learning methods, and in 2008, he obtained a Ph.D. degree in Computer Science from the University of Freiburg. He then worked for 4 years as a post-doctoral researcher at the Idiap Research Institute in Martigny, Switzerland, in the field of computer vision and mainly face tracking. As of today, Stefan Duffner is an associate professor in the IMAGINE team of the LIRIS research lab at the National Institute of Applied Sciences (INSA) of Lyon, France.

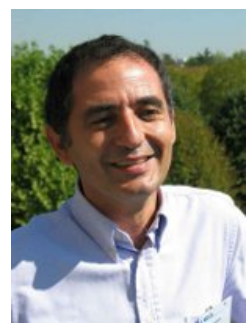

Khalid Idrissi received a B.S. degree and the M.S in 1984 in the school of electrical engineering from INSA-Lyon, France. From 1985 to 1991 , he has been working as an engineer, then as project leaders in industry. He received the "Agrégation" in electrical engineering in 1994 and he has been "professeur agrégé" until 2003 at the French Guyana University then at INSALyon. He received his Ph.D degree in 2003, and then "HDR" in 2011. He is currently working as an Associate Professor at the Telecommunication Department of INSA-Lyon since 2004. He is mainly working on image analysis and segmentation for image compression, image retrieval, shape detection and identification, facial analysis.

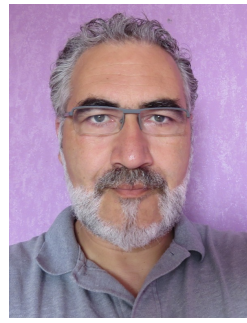

Christophe Garcia received his Ph.D. degree in computer vision from Université Claude Bernard Lyon I, France, in 1994 and his Habilitation à Diriger des Recherches (HDR) from INSA Lyon I University of Lyon I, in 2009. Since 2010, he is a Full Professor at INSA de Lyon and the deputy Director of the LIRIS laboratory. He holds 17 industrial patents and has published more than 140 articles in international conferences and journals. He has served in more than 30 program committees of international conferences and is an active reviewer in 15 international journals where he has coorganized several special issues. His current technical and research activities are in the areas of deep learning, neural networks, pattern recognition and computer vision.

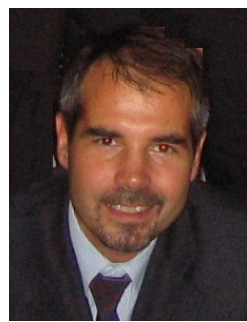

Atilla Baskurt received the B.S. degree in 1984 the M.S. in 1985 and the Ph.D. in 1989, all in electrical engineering from INSA-Lyon, France. From 1989 to 1998, he was "Maître de Conférences" at INSA-Lyon. Since 1998, he is Professor in Electrical and Computer Engineering, first at the University Claude Bernard of Lyon, and now at INSA-Lyon. From 2003 to 2008, he was the Director of the Telecommunication Department of INSA-Lyon. From September 2006 to December 2008, he was "Chargé de mission" on Information and Communication Technologies (ICT) at the French Research Ministry MESR. Currently, he is Director of the LIRIS Research Lab. He leads his research activities in two teams of LIRIS: the IMAGINE team and the M2DisCo team. These teams work on image and 3D data analysis and segmentation for image compression, image retrieval, shape detection and identification. His technical research and experience include digital image processing, 2D-3D data analysis for segmentation, compression and retrieval, video content analysis for action recognition and object tracking. 